\title{
Difficult diagnosis and surgical treatment of acute aortic syndrome
}

\author{
Wioleta Kurpińska-Kac ${ }^{1}$, Jakub Osowski ${ }^{1}$, Natalia Sobczyk ${ }^{1}$, Andrzej Łoś ${ }^{2}$ \\ ${ }^{1}$ Students' Scientific Circle Cardiac and Vascular Surgery Department, Medical University of Gdansk, \\ Gdansk, Poland \\ ${ }^{2}$ Cardiac and Vascular Surgery Department, Medical University of Gdansk, Gdansk, Poland
}

Kardiochirurgia i Torakochirurgia Polska 2021; 18 (4): 265-267

Acute aortic syndrome (AAS) continues to be a major clinical problem due to the need for urgent diagnosis and complex surgical management. We present a case that was not only surgically demanding, but also caused many difficulties in the preoperative diagnostic process. Typical clinical symptoms of AAS did not correlate with imaging study results, including computed tomography (CT) angiography and transthoracic echocardiography (TTE).

A 64-year-old female patient was admitted to the hospital due to tearing, excruciating chest pain lasting for 18 hours and radiating to the interscapular area. Pain tended to slightly decrease with time. Other symptoms were transient numbness of the left lower extremity (a few hours) and amblyopia, lasting for several minutes. She had a history of poorly controlled hypertension, nicotinism, nephrectomy due to renal cell carcinoma, peptic ulcer disease, anxiety, depressive disorder, and addiction to clonazepam.
The patient was transferred from the district hospital, where the CT angiography was performed. This imaging study showed thickening of the ascending aortic wall, so acute aortic dissection and/or intramural hematoma of the ascending aorta were suspected. Laboratory tests showed slightly increased D-dimers and negative troponins. ECG was within the normal limits, and no signs of acute myocardial infarction were noted.

During the 5-hour stay at the intensive care unit, higher-resolution CT angiography was performed (Figure $1 \mathrm{~A}$ ), which ruled out aortic dissection and suggested giant-cell arteritis, due to the thickened wall of the descending, ascending and thoracic aorta. No obvious false lumen or entry site was found, and the true lumen was smoothly round on the course of the aorta. Ascending aorta diameter was $5 \mathrm{~cm}$. However, the thickened wall could represent an intramural hematoma, but the radiologist describing the imaging study considered giant-cell arteritis more plausible.
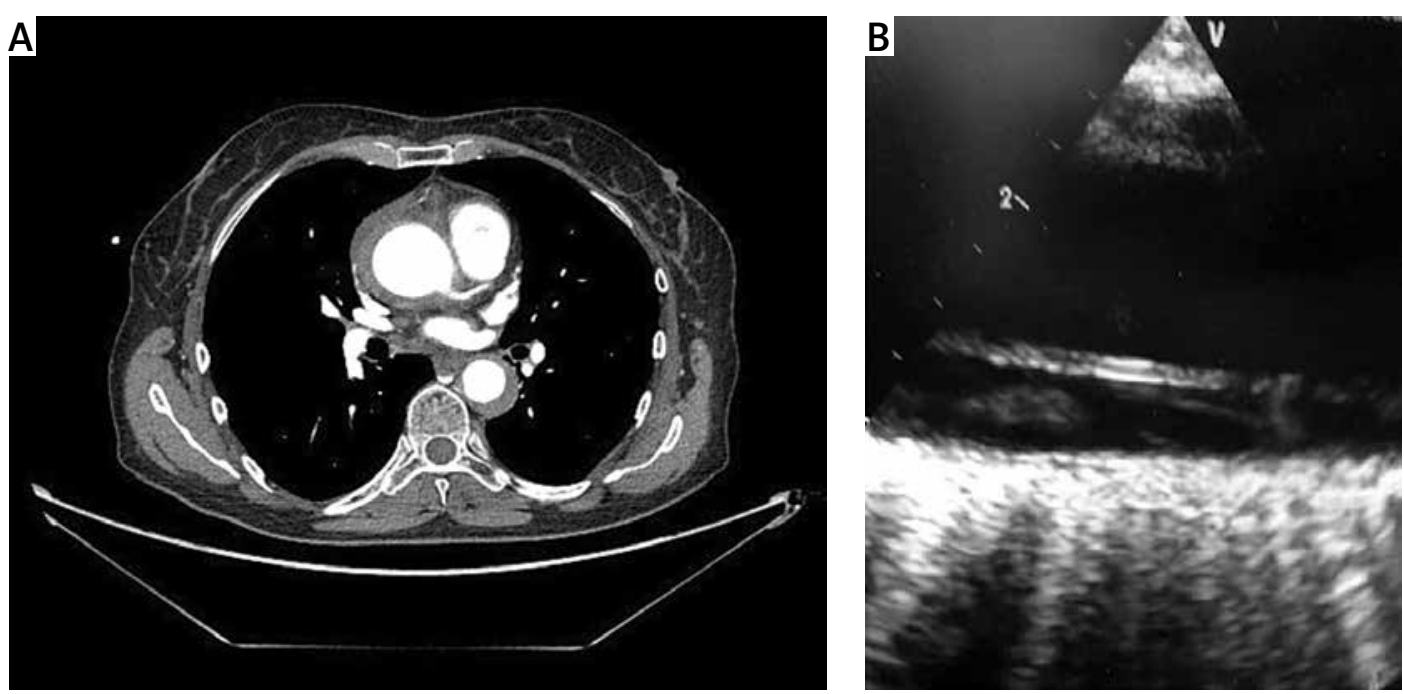

Figure 1. A - High resolution CT angiography - thickened wall of asceding $(4 \mathrm{~mm})$ and descending $(7 \mathrm{~mm})$ aorta suggesting giant-cell arteritis. No visible false lumen or entry site was found, the aortic lumen was smoothly round on its course. B - Preoperative TEE - false lumen (bottom) clearly visible in ascending aorta

Address for correspondence: Wioleta Kurpińska-Kac, Students' Scientific Circle Cardiac and Vascular Surgery Department, Medical University of Gdansk, Gdansk, Poland, e-mail: w.kurpinskakac@wp.pl

Received: 27.05.2021, accepted: 19.09.2021. 

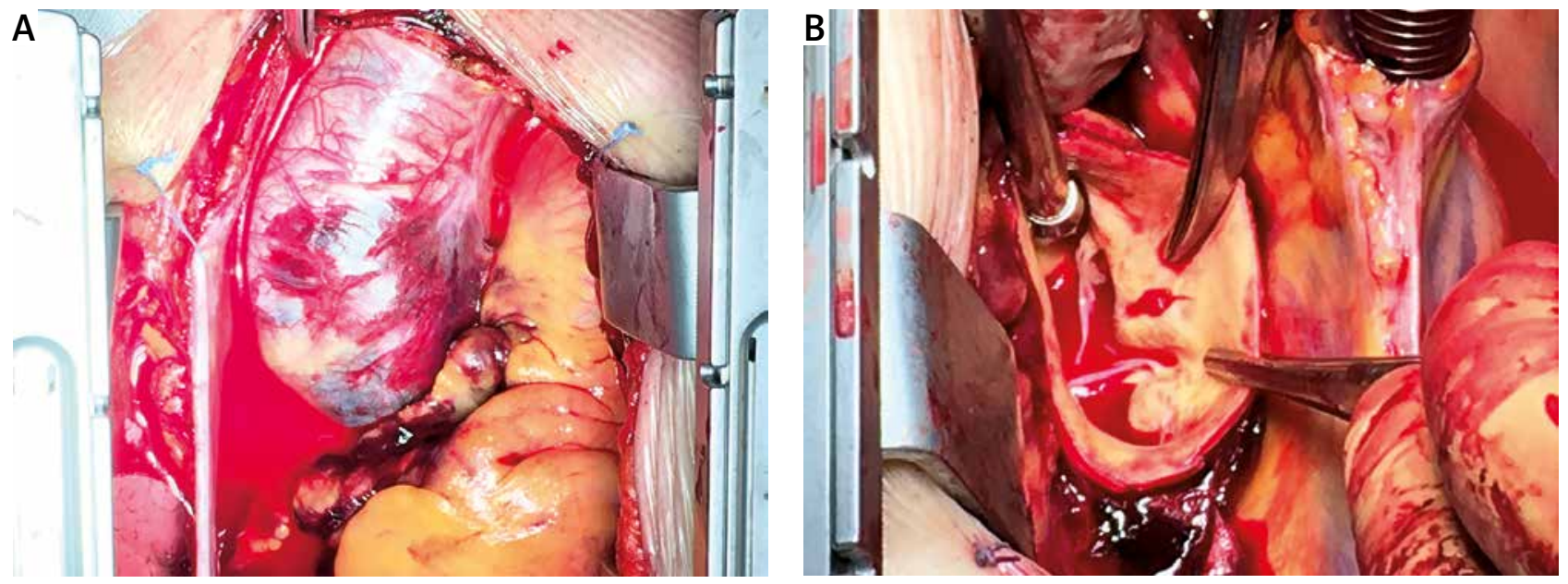

Figure 2. A - Hematoma of ascending aorta seen after median sternotomy. B - Entry site in non-coronary Valsalva sinus

No targeted coronary artery CT was performed, but the aortic angiography revealed the approximate condition of coronary arteries - no obvious pathology was found. It must be noted that in this case coronary CT (and calcium score) should be performed - even if the acute coronary syndrome was ruled out, the patient still had significant risk of chronic coronary syndrome.

After the careful consideration of benefits and risk, the decision to perform exploratory sternotomy was made. There was no clearly confirmed AAS, and angio-CT could only slightly suggest the presence of intramural hematoma (IMH). For this reason, it was concluded that the risk of exploratory sternotomy is lower than the risk of possible progression of unrecognized AAS. Just before the surgery, while the patient was anesthetized on the surgical table, the TEE was performed. In our unit, TEE has been used routinely for several years; due to extensive experience, complications are rare. The formation of a false lumen in the ascending aorta was noted (Figure $1 \mathrm{~B}$ ), which confirmed the presence of aortic dissection and need for urgent surgery. After typical median incision, the pericardial sac was opened and a large hematoma of the whole ascending aorta was visualized (Figure $2 \mathrm{~A}$ ). On cardiopulmonary bypass, a slight intimal rupture was found just above the non-coronary sinus of aorta (Figure 2 B). Intraoperative findings were diagnostic for acute aortic dissection. The aortic wall was normal in macroscopic examination; therefore a tissue sample for pathological study was not obtained. The site of entry rupture was repaired with tissue glue and the ascending aorta was replaced with a Dacron vascular graft. The patient's condition was serious, and there was a major risk of acute postoperative complications such as multi-organ failure; therefore the longer and more technical demanding aortic valve reimplantation procedure was abandoned in favor of ascending aortic replacement and repairing the entry site. The postoperative period was uneventful. During the follow-up after 3 months after the surgery no significant abnormalities were found.

Acute aortic syndrome is an emergency condition. This triad of high-mortality diseases include aortic dissection
(AD), IMH and penetrating aortic ulcer (PAU) [1]. The most common is $A D$, in which the continuity of the intimal membrane is disrupted, resulting in dissection of the middle arterial layer by flowing blood. This is called the false lumen. $\mathrm{IMH}$ is a disease in which the hematoma grows within the middle layer of the aortic wall, while the intimal layer is unaffected. The pathogenesis of this process is not understood clearly; the diagnosis of IMH is difficult and burdened with numerous errors. Most IMHs found in the imaging studies are ADs with a clotted false lumen. However, IMH has different clinical presentation, characteristics, demographics and treatment strategies, which may be ethnically or genetically based. PAU is ulceration in atherosclerotic plaque in the aorta that penetrates from the intimal membrane to the medial layer of the arterial wall. This form of AAS is very rare, but it is a significant clinical problem, even if asymptomatic, as progression of ulceration may lead to AD, IMH, pseudoaneurysm or aortic rupture.

Treatment of AAS type A is surgical and should be performed immediately to reduce patient mortality. CT angiography remains the gold diagnostic standard for these diseases. The most common symptom of acute aortic syndrome is chest pain [2]. However, chest pain may occur in a variety of chest diseases, which can cause difficulties in the diagnostic process. In the literature, there are cases of AD misdiagnosed as stent thrombosis [3] or acute myocardial infarction (AMI). It should be noted that approximately $7 \%$ of patients with AD have concomitant AMI [4]. AAS could also appear in an unusual form, such as circular dissection, first described by Hufnagel and Conrad in the 1960s [5]. Therefore, a thorough differential diagnosis should be performed. AAS is more commonly caused by a sudden increase of blood pressure. This condition should be suspected in patients with poorly controlled hypertension or in patients abusing stimulants, which could cause a sudden increase of blood pressure (there is a case of acute aortic dissection (AAD) caused by mephedrone in a young patient [6]). Other risk factors are connective tissue disorders (such as Marfan's syndrome), history of cardiac surgery or aortic diseases [2]. It must be noted at this point that the majority 
of aortic pathologies and diseases could lead to acute aortic syndrome. One of these conditions is giant-cell arteritis, which was suspected in this patient after the second CT angiography.

This case reminds us how important it is to collect a detailed history from the patient. In the modern era there are many types of radiological studies. Undoubtedly, they allow for better diagnosis and visualization of structures previously not accessible for examination. However, the situation where there are only minor changes, sometimes even insufficient to make a clear diagnosis, could be possible. Thus, only the combination of novel imaging techniques, a thoroughly collected patient history and physical examination allows for a proper diagnosis and clinical success.

\section{Disclosure}

The authors report no conflict of interest.

\section{References}

1. Corvera JS. Acute aortic syndrome. Ann Cardiothorac Surg 2016; 5: 188-193.

2. Erbel R, Aboyans V, Boileau C, Bossone E, Di Bartolomeo R, Eggebrecht H, Evangelista A, Falk V, Frank H, Gaemperli O, Grabenwöger M, Haverich A, lung B, Manolis AJ, Meijboom F, Nienaber CA, Roffi M, Rousseau H, Sechtem U, Sirnes PA, von Allmen RS, Vrints CJM, ESC Committee for Practice Guidelines. 2014 ESC Guidelines on the diagnosis and treatment of aortic diseases. Eur Heart J 2014; 35: 2873-2926.

3. Rzepa $\measuredangle$, Walczewski M, Fojt A, Kowalik R. Suspected case of subacute stent thrombosis referred for coronary angiography turns out to be unusual aortic dissection presentation. Kardiochir Torakochir Pol 2020; 17: 44-46.

4. Wang D, Zhang LL, Wang ZY, Zhang ZY, Wang Y. The missed diagnosis of aortic dissection in patients with acute myocardial infarction: a disastrous event. J Thorac Dis 2017; 9: 636-639.

5. Perek $B$, Juszkat R, Frankiewicz $M$, Jemielity $M$. Very rare case of type $A$ acute aortic dissection. Kardiochir Torakochir Pol 2011; 8: 49-51.

6. Barwina M, Zając M, Lango R, Betlejewski P, Waldman W, Sein Anand J. Acute aortic dissection due to intoxication with mephedrone - a case report. Kardiochir Torakochir Pol 2012; 9: 378-382. 